\title{
Validation of a Postoperative Delirium Prediction Model
}

\author{
Ashok Biju¹ B.S., Babar Khan² M.D. M.S., Heidi Lindroth² Ph.D. R.N. \\ ${ }^{1}$ Indiana University School of Medicine, ${ }^{2}$ Indiana University School of Medicine, \\ Department of Internal Medicine.
}

Background and Hypothesis: A prediction model utilizing the National Surgical Quality Improvement Program risk calculator for serious complications (NSQIP$\mathrm{SC}$ ) and measures of cognitive function has been demonstrated to predict postoperative delirium incidence and severity in patients after non-cardiac surgery. The next step is to test this model's generalizability by validating in different patient populations and evaluating the robustness of the model with and without parameters of cognition. We hypothesized that the prediction model would still function in predicting postoperative delirium in patients undergoing major thoracic surgery.

Experimental Design or Project Methods: A secondary data analysis of a randomized clinical trial involving 135 individuals who underwent major thoracic surgery from October 2013 to June 2015 was done. Delirium incidence and severity were tracked postoperatively through the Confusion Assessment Method for the ICU (CAM-ICU) and the Delirium Rating Scale-R-98 (DRS), respectively. The Repeatable Battery for the Assessment of Neuropsychological Status (RBANS) was used to assess preoperative cognition. Linear regressions and specificity/sensitivity calculations were performed to evaluate the model.

Results: One hundred and thirty-two patients with a mean age of $59 \pm 13$ years, $33 / 132$ CAM-ICU+, and a mean DRS peak of 3.12 \pm 3.43 were analyzed. Linear regression of NSQIP-SC scores moderately predicted delirium severity $(P<0.001$, Adjusted $R^{2}$ : 0.28 ). Addition of a cognitive measure did not significantly improve the model $\left(P<0.001\right.$, Adjusted $R^{2}$ : 0.29). NSQIP-SC moderately predicted delirium incidence (sensitivity: $60.61 \%$, specificity: $68.69 \%$, AUROC: 0.73 ). Addition of a cognitive measure (sensitivity: $61.54 \%$, specificity: $68.69 \%$, AUROC: 0.73 ) did not significantly improve the model.

Conclusion and Potential Impact: NSQIP-SC was a predictor variable for delirium incidence and severity. Addition of a cognitive measure did not have any appreciable effect on the prediction model. Further expanding the scope of the model can grant clinicians a tool to identify patients at risk for developing postoperative delirium. 